\title{
Hyperbolic groups which fiber in infinitely many ways
}

\author{
TARALEE MECham \\ ANTARA MUKHERJEE
}

\begin{abstract}
We construct examples of CAT(0), free-by-cyclic, hyperbolic groups which fiber in infinitely many ways over $\mathbb{Z}$. The construction involves adding a specialized square 2-cell to a non-positively curved, squared 2-complex defined by labeled oriented graphs. The fundamental groups of the resulting complexes are CAT(0), hyperbolic, free-by-cyclic and can be mapped onto $\mathbb{Z}$ in infinitely many ways.
\end{abstract}

20F65; $51 \mathrm{H} 99$

\section{Introduction}

Topologists have been interested in fiberings of 3-manifolds since the early 1960's. A 3-manifold is said to fiber over $S^{1}$ if it is the mapping torus of a surface. A key to relating this topological fibering to the notion of algebraic fibering was expressed by Stallings in 1962 [15]. He proved the following:

Theorem For an irreducible compact 3-manifold, $M^{3}$, if $\pi_{1}\left(M^{3}\right)$ has a finitely generated, normal subgroup $G$ (which is not $\mathbb{Z}_{2}$ ), whose quotient group is $\mathbb{Z}$, then $M^{3}$ fibers over $S^{1}$. The fiber here is a 2 -manifold $T$ embedded in $M^{3}$, such that $\pi_{1}(T)=G$.

The fundamental groups of these manifolds are a special class of HNN-extensions that extend the fundamental group of the surface by $\mathbb{Z}$.

In 1969, Tollefson showed [17] that there were 3-manifolds that fibered over $S^{1}$ in infinitely many ways. From the group theory perspective, this means that the fundamental groups of these 3-manifolds can be expressed in infinitely many ways as surface-by-cyclic groups with the genus of the surface going to infinity.

In 1976 D A Neumann [13] proved the following:

Theorem If $M$ is a 3-manifold, which is a total space of a fiber bundle with base $S^{1}$, and the fiber is a closed $2-$ manifold $F$ (genus $(F) \geqslant 2$ for $F$ orientable and 
genus $(F) \geqslant 3$ for $F$ non-orientable), then $M$ has a unique fibering over $S^{1}$ iff $\operatorname{rank}\left(H_{1}(M ; \mathbb{Z})\right)=1$. In the case that $\operatorname{rank}\left(H_{1}(M ; \mathbb{Z})\right) \neq 1, M$ fibers over $S^{1}$ with fiber any of infinitely many distinct closed surfaces.

A manifold has a unique fiber over $S^{1}$, according to Neumann if, for any fibering of $M$ over $S^{1}$ with fiber $F^{\prime}$, we have $F \cong F^{\prime}$.

In 1998 Thurston [16] proved that every atoroidal three-manifold that fibers over the circle has a hyperbolic structure. Using branched covers of the 3-torus, in 2006 Hilden, Lozano and Montesinos-Amilibia [10] constructed explicit examples of hyperbolic manifolds which have infinitely many fibrations over $S^{1}$.

In 2007 Jack Button [6] proved the following result:

Theorem Given a finitely presented group with Betti number at least two such that $G$ admits a homomorphism onto $\mathbb{Z}$ with finitely generated kernel and the Alexander polynomial of $G$ is non-constant then, $G$ admits homomorphisms onto $\mathbb{Z}$ that have finitely generated kernels with arbitrarily high Betti numbers.

He also shows that if $G$ has Betti number at least three, then $G$ admits a homomorphism onto $\mathbb{Z}$ with finitely generated kernel. Another application of this theorem is that a free-by-cyclic group with Betti number at least two, fibers infinitely over the circle.

The main theorem of this paper produces some results in the context of $\operatorname{CAT}(0)$, hyperbolic, free-by-cyclic groups.

Theorem 1.1 There exist CAT(0), hyperbolic, free-by-cyclic groups which have infinitely many epimorphisms onto $\mathbb{Z}$. Furthermore, each of these groups is isomorphic to $F_{n} \rtimes \mathbb{Z}$ for infinitely many $n$.

We prove Theorem 1.1 by constructing groups that are hyperbolic, free-by-cyclic and map onto $\mathbb{Z}^{2}$. These groups are the fundamental groups of specially constructed nonpositively curved, squared 2-complexes. In their 1999 paper [12], Ian Leary, Graham Niblo and Dani Wise constructed finitely generated groups which are free-by-cyclic and contain a non-free, locally free subgroup. The smallest such example found by them is $G \cong F_{n} \rtimes \mathbb{Z}$ for $n=3$. In addition, they have examples for larger values of $n$ that are word hyperbolic using Bestvina-Brady Morse theory.

The paper is organized as follows: Section 2 gives some background about nonpositively curved squared 2 -complexes, $\delta$-hyperbolic spaces, and Morse theory necessary for the construction of the groups. Section 3 gives a description of the building 
blocks for the construction in Section 4 where we give the actual construction of groups with reducible monodromy automorphism, proving Theorem 1.1. Section 5 provides an alternative construction to produce an example of a group which may have an irreducible automorphism. This construction does not use the building blocks from Section 3. We conclude the paper with Section 6, where we present some questions that are yet to be answered.

This paper would not have been possible without the support and guidance of our advisor, Dr Noel Brady.

\section{Background and definitions}

Theorem 1.1 requires that we produce free-by-cyclic, hyperbolic groups, so we will recall some facts about $\delta$-hyperbolic spaces and combinatorial Morse theory in this section. Since the groups we construct are the fundamental groups of non-positively curved, squared 2-complexes, we first recall some facts about non-positively curved spaces. We then quote Gromov's Flat Plane Theorem in [9] which helps us show that the groups are indeed hyperbolic. Finally, we will present some facts about Morse Theory and conclude the section with Proposition 2.10 which we will use to both prove that our groups are free-by-cyclic and compute the free rank of the kernel for various maps onto $\mathbb{Z}$.

We start with some definitions associated with non-positively curved spaces. These definitions can be found in Bridson and Haefliger's book [5].

Definition 2.1 A piecewise Euclidean 2-complex $K$ is a 2-complex obtained from a collection of convex cells in the Euclidean plane by identifying their edges via isometries.

Definition 2.2 The link of a vertex, $v$, in a convex 2-cell $c$ (denoted by $\operatorname{Lk}(v, c)$ ) of a 2-complex, is defined as the collection of unit tangent vectors to the space at $v$ which point to $c$. This is a spherical cell of dimension $(n-1)$.

Suppose there are two convex 2-cells $c, c^{\prime}$ with vertices $v, v^{\prime}$ respectively which are identified along the faces via an isometry $f$ which identifies $v$ with $v^{\prime}$. The derivative of $f$ gives a spherical isometry which identifies a face of $\operatorname{Lk}(v, c)$ and a face of $\operatorname{Lk}\left(v^{\prime}, c^{\prime}\right)$. Thus, the the link of a vertex in a 2-complex is naturally described as a piecewise spherical complex. 
Definition 2.3 The link of a vertex satisfies the large link condition if it has no circuits of length $<2 \pi$. This definition is the same as saying that the link satisfies the no empty triangles condition or equivalently, the link is a flag complex. See [5] for more details. It is important to notice here that this definition is only true for square complexes.

Definition 2.4 A piecewise Euclidean 2-complex $K$ is said to be non-positively curved if $\operatorname{Lk}(v, K)$ is large (that is, no circuits of length less than $2 \pi$ ) for all $v \in K^{(0)}$. In this context a link is a simplicial complex, which implies it has no edge loops and no bigons.

The following is a theorem stated by Gromov [9], which was later proved by Bridson [4]. We will use this theorem to show that the universal covers of the 2-complexes we construct are hyperbolic; therefore, the corresponding fundamental groups are hyperbolic. This result is analogous to Eberlein's [7] earlier work, where he showed that: A compact non-positively curved Riemannian manifold either has a word-hyperbolic fundamental group or its universal cover contains an isometrically embedded copy of the Euclidean plane.

Theorem 2.5 (The Flat Plane Theorem [5]) A proper cocompact CAT(0) space is hyperbolic if and only if it does not contain a subspace isometric to $\mathbb{E}^{2}$.

We will also need to ensure that our groups are free-by-cyclic. We will use combinatorial Morse theory to establish this fact. Following are the basic definitions necessary for this method of proof. These definitions, and proofs for the theorems, can be found in [1].

Definition 2.6 A finite-dimensional cell-complex $X$ is said to be an affine cell-complex if it has the following structure. An integer $m>\operatorname{dim}(X)$ is given, and for each cell $e$ of $X$ we are given a convex polyhedral cell $C_{e} \subset \mathbb{R}^{m}$ and a characteristic function $\chi_{e}: C_{e} \rightarrow e$ such that the restriction of $\chi_{e}$ to any face of $C_{e}$ is a characteristic function of another cell, possibly pre-composed by a partial affine homeomorphism of $\mathbb{R}^{m}$.

In our construction, our highest dimensional cell will be two-dimensional. In fact, the complexes will be made of Euclidean squares identified along the edges, so they will be affine.

Definition 2.7 A map $f: X \rightarrow \mathbb{R}$ defined on an affine cell-complex $X$ is a Morse function if:

- for every cell $e$ of $X, f_{\chi_{e}}: C_{e} \rightarrow \mathbb{R}$ extends to an affine map $\mathbb{R}^{m} \rightarrow \mathbb{R}$ and $f_{\chi_{e}}$ is constant only when $\operatorname{dim}(e)=0$, and 
- the image of the 0 -skeleton is discrete in $\mathbb{R}$.

One can think of a Morse function as a height function where each cell of the complex has a unique maximum and a unique minimum. Since we want to show that our groups are free-by-cyclic, we will be interested in maps from our complexes to the circle. The following definition relates these maps to Morse functions.

Definition 2.8 A circle-valued Morse function on an affine cell complex $X$ is a cellular map $f: X \rightarrow S^{1}$, with the property that $f$ lifts to a Morse function between universal covers. When the context is clear, we will simply refer to these as Morse functions.

The links of the vertices of a 2-complex are graphs. It turns out that in the presence of a Morse function, certain subgraphs of the link give us important information about the structure of the fundamental group of the 2-complex. The following definition and proposition describe this relationship.

Definition 2.9 Suppose $X$ is an affine cell complex and $f: X \rightarrow S^{1}$ is a circle-valued Morse function. Choose an orientation of $S^{1}$, which lifts to an orientation of $\mathbb{R}$, and lift $f$ to a map of universal covers $\tilde{f}: \tilde{X} \rightarrow \mathbb{R}$. Let $v \in X^{(0)}$ and note that the link of $v$ in $X$ is naturally isomorphic to the link of any lift $\tilde{v}$ of $v$ in $\tilde{X}$. We say that a cell $\tilde{e} \subset \tilde{X}$ contributes to the ascending (respectively descending) link of $\widetilde{v}$ if $\widetilde{v} \in \widetilde{e}$ and only if $\left.\widetilde{f}\right|_{\tilde{e}}$ achieves its minimum (respectively maximum) value at $\tilde{v}$. The ascending (respectively descending) link of $v$ is then defined to be the subset of $\operatorname{Lk}(v, X)$ naturally identified with the ascending (respectively descending) link of $\widetilde{v}$.

The following proposition is an important result of Morse theory and helps us check that the fundamental groups of the 2-complexes we construct are indeed free-by-cyclic and gives us a formula for determining the free rank of the groups.

Proposition 2.10 (Free-by-Cyclic [1]) If $f: X \rightarrow S^{1}$ is a circle-valued Morse function on the 2-complex $X$ all of whose ascending and descending links are trees, then $X$ is aspherical, and $\pi_{1}(X)$ is free-by-cyclic. In fact, $\pi_{1}(X)=F_{k} \rtimes \mathbb{Z}$ where $k=1-\chi\left(f^{-1}(p)\right)$ for $p \in S^{1}$.

\section{Building blocks}

The groups that we construct in Section 4 are one-relator quotients of free products of some building block groups. In this section we describe these building block groups and show that they are the fundamental groups of non-positively curved 2-complexes. We also show that these groups are hyperbolic and free-by-cyclic. 
Remark 3.1 We will be looking at 2-complexes which have a unique vertex for the rest of the paper.

We start this section by defining a Labeled Oriented Graph (LOG). The group presentation corresponding to a particular LOG is used as the building block group for the example constructed in Section 4. The following definition is taken from [1].

Definition 3.2 A labeled oriented graph or LOG, consists of a finite, directed graph with distinct labels on all the vertices and oriented edge labels taken from the set of vertex labels. An LOG defines a finite group presentation in the following manner. The set of generators is in 1-1 correspondence with the set of vertex labels and the set of relators is in 1-1 correspondence with the set of edges, so that an edge labeled $a$ oriented from vertex $u$ to vertex $v$ corresponds to a conjugation relation $a v a^{-1}=u$. In the case that the graph is a tree, we call it a labeled oriented tree or LOT, and call the corresponding finite presentation an LOT presentation.

Howie [11] was one of the first people to define and study the group presentations of LOTs.
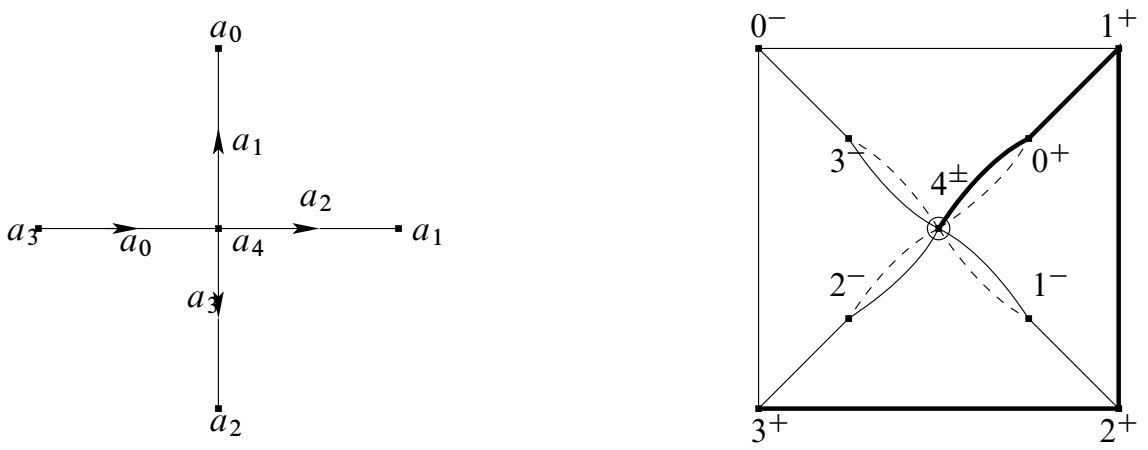

Figure 1: The LOT presentation of $L_{\left(a_{i}\right)}$ and the link of $v$ in $X_{L_{\left(a_{i}\right)}}$. The circle in the center of the link represents the points $4^{+}$and $4^{-}$. The dashed lines are edges connecting to $4^{-}$. There is no edge connecting $4^{+}$to $4^{-}$. The descending link is shown in bold.

Remark 3.3 The main component of our construction is an LOT group with five generators, $a_{0}, \ldots, a_{4}$, denoted by $L_{\left(a_{i}\right)}$. Figure 1 shows the graph of the LOT. The corresponding presentation 2-complex $X_{L_{\left(a_{i}\right)}}$ consists of a single vertex, a bouquet of five circles, and four square 2-cells. The group presentation of this complex is given by

$$
L_{\left(a_{i}\right)}=\left\langle a_{0}, a_{1}, a_{2}, a_{3}, a_{4} \mid a_{4}=a_{0}^{a_{1}}, a_{4}=a_{1}^{a_{2}}, a_{4}=a_{2}^{a_{3}}, a_{3}=a_{4}^{a_{0}}\right\rangle
$$


where $a_{i}^{a_{j}}=a_{j} a_{i} a_{j}^{-1}$.

The link of the vertex of the corresponding presentation 2-complex is also shown in Figure 1. Since $X_{L_{\left(a_{i}\right)}}$ is a piecewise Euclidean 2-complex, it is easy to see that the link is large and hence, the universal cover $\tilde{X}_{L_{\left(a_{i}\right)}}$ is CAT(0). In order to show that $L_{\left(a_{i}\right)}$ is hyperbolic we will need to use the Flat Plane Theorem. The following definition will be useful in its application.

Definition 3.4 A poison corner is a corner of a 2-cell for which the corresponding edge in the link is not a part of any length-four circuit.

Remark 3.5 This definition is helpful to us because the 2-cells of the presentation 2-complex $X_{L_{\left(a_{i}\right)}}$ are Euclidean square 2-cells. This means that all edges in the link are of length $\pi / 2$. Hence, any square of our complex that contains a poison corner cannot be used to develop a flat plane in the universal cover.

Theorem 3.6 The universal cover of $X_{L_{\left(a_{i}\right)}}$ has no isometrically embedded flat planes.

Proof The link has no bigons or empty triangles, so the link is large. We now need only ensure that no planes develop in the universal cover. There are four edges of the link that act as bridges from the inner suspension to the outer circuit of the link. They are

$$
a_{1}^{+} a_{0}^{+}, \quad a_{2}^{+} a_{1}^{-}, \quad a_{3}^{+} a_{2}^{-}, \quad a_{0}^{-} a_{3}^{-} .
$$

If we remove these four edges, the link reduces to two separate components. The outer component is the length-four circuit $a_{0}{ }^{-} a_{1}+a_{2}{ }^{+} a_{3}{ }^{+} a_{0}{ }^{-}$, and the combinatorial distance between any two vertices of this component is at least 1 . On the other hand, the inner component is the suspension of the two vertices $a_{4}{ }^{+}$and $a_{4}{ }^{-}$on the set vertices $a_{0}{ }^{+}, a_{1}{ }^{-}, a_{2}{ }^{-}$, and $a_{3}{ }^{-}$. The distance between any pair of these four vertices in the inner component is at least 2 . Therefore, any circuit containing one of these bridges has to be of length at least 5 . Hence, the bridges represent poison corners.

Any isometrically embedded flat plane is tiled by a collection of the 2-cells of the

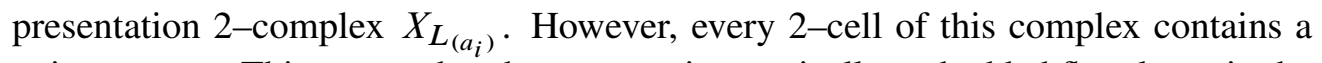
poison corner. This means that there are no isometrically embedded flat planes in the universal cover of the complex.

Corollary 3.7 $L_{\left(a_{i}\right)}$ is a hyperbolic group.

Proof The universal cover of the 2-complex $X_{L_{\left(a_{i}\right)}}$ is CAT(0) since the link is large and Theorem 3.6 further implies that the universal cover $\tilde{X}_{L_{\left(a_{i}\right)}}$ has no isometrically embedded flat planes, so using the Flat Plane Theorem, we know that $\tilde{X}_{L_{\left(a_{i}\right)}}$ is $\delta-$ hyperbolic and the corresponding group $L_{\left(a_{i}\right)}$ is hyperbolic. 
Now we will show that $L_{\left(a_{i}\right)}$ is also free-by-cyclic. The proof of the following proposition is along the same lines as the first part of the proof of [1, Theorem 3].

Proposition 3.8 $L_{\left(a_{i}\right)}$ is free-by-cyclic and is isomorphic to $F_{4} \rtimes \mathbb{Z}$.

Proof The presentation 2-complex $X_{L_{\left(a_{i}\right)}}$ of the group $L_{\left(a_{i}\right)}$, admits a circle-valued Morse function which is defined by mapping the vertex to the base vertex of the circle and each directed edge is mapped once around the target circle. The same map extends linearly over the 2-cells of $X_{L_{\left(a_{i}\right)}}$. The descending link of this Morse function is shown in bold on the link in Figure 1. Since both the ascending and descending links are trees, Proposition 2.10 implies that the LOT group is free-by-cyclic. The pre-image of the vertex in the target circle under the Morse function is a bouquet of four circles, so the group is isomorphic to $F_{4} \rtimes \mathbb{Z}$.

Although $L_{\left(a_{i}\right)}$ is hyperbolic and free-by-cyclic, it only fibers in one way over $\mathbb{Z}$ because the relations of the group are all conjugation relations. This means that the image of all the generators will be the same under any map to $\mathbb{Z}$. Therefore there is only one nontrivial map to $\mathbb{Z}$, up to post composition with a monomorphism of $\mathbb{Z}$.

Remark 3.9 It is easy to see that for any LOT group represented by a tree with $k+1$ vertices, a single valence $k$ vertex, and with edge and vertex labels permuted as in the $L_{\left(a_{i}\right)}$ case, the previous theorems hold. In fact, the link will consist of an outer $k$-gon with $k$ bridges to $k$ inner vertices and the suspension of these inner vertices on the vertices $a_{k}^{+}$and $a_{k}^{-}$. This means that the group will be hyperbolic and isomorphic to $F_{k} \rtimes \mathbb{Z}$. For arbitrary $k \geqslant 4$, we will denote the group and the presentation 2-complex associated to it by $L_{\left(k, a_{i}\right)}$, and $X_{L_{\left(k, a_{i}\right)}}$ respectively.

\section{The construction of reducible examples}

Now we will use the LOT groups from the previous section to prove Theorem 1.1. We will begin by focusing on the topological aspects of our construction and show that the groups produced are free-by-cyclic and map onto $\mathbb{Z}^{2}$. This will be the content of Theorem 4.2 below. Since $\mathbb{Z}^{2}$ has infinitely many nontrivial maps onto $\mathbb{Z}$, by showing that a free-by-cyclic group maps onto $\mathbb{Z}^{2}$, we get infinitely many maps from this group onto $\mathbb{Z}$ for free. Next we will address the geometry of our particular examples by showing that they are indeed hyperbolic, thus providing the proof of Theorem 1.1. We will then give a method for computing the free rank of the kernel of a map from our groups onto $\mathbb{Z}$ in Lemma 4.3. We conclude this section by writing down an explicit (reducible) automorphism for the group produced in the proof of Theorem 1.1. . 
Remark 4.1 Since all of the spaces that we deal with have a single vertex, we shall denote the fundamental group element corresponding to any 1-cell say $x$, of a 2complex, by $[x]$. If $f$ is the map from a presentation 2-complex, $K$, to a circle and $f_{*}$ the map between the corresponding fundamental groups, that is, $f_{*}: \pi_{1}(K) \rightarrow \mathbb{Z}$, then using the notation given above, for Theorem 4.2 we will define $\alpha=f_{*}([a])$ and $\beta=f_{*}([b])$.

Theorem 4.2 Given 2-complexes $K_{j}$ for $j=1,2$ satisfying:

(i) $\left|K_{j}^{(0)}\right|=1$,

(ii) there exist Morse functions $f_{j}: K_{j} \rightarrow S^{1}$ such that the ascending and descending links are trees,

(iii) there are 1-cells $a_{0}, a_{1} \in K_{1}$ and $b_{0}, b_{1} \in K_{2}$ such that $\left|\alpha_{0}\right|+\left|\beta_{1}\right|=\left|\alpha_{1}\right|+\left|\beta_{0}\right|$, then the 2-complex, $K$, obtained from $K_{1} \sqcup K_{2} /\left(K_{1}^{(0)} \sim K_{2}^{(0)}\right)$ by attaching a square 2-cell according to the relation $r=a_{0}^{ \pm 1} b_{1}^{ \pm 1} a_{1}^{\mp 1} b_{0}^{\mp 1}$, satisfies:

(i) $\pi_{1}(K)$ is free-by-cyclic and,

(ii) $\pi_{1}(K)$ maps onto $\mathbb{Z}^{2}$.

Proof (i) We start by labeling the unique vertex of $K_{j}$ by $v_{j}$ for $j=1,2$ and consider the 2-complex $K_{1} \vee K_{2}=K_{1} \sqcup K_{2} / v_{1} \sim v_{2}$, calling the common vertex $v$. The fundamental group of this complex is the free product of the fundamental groups of $K_{1}$ and $K_{2}$. By the functorality of the wedge product, $f_{1}$ and $f_{2}$ induce the following map:

$$
f_{1} \vee f_{2}: K_{1} \vee K_{2} \rightarrow S^{1} \vee S^{1}
$$

Using $f_{1} \vee f_{2}$, we define a circle-valued Morse function, $\operatorname{pr} \circ\left(f_{1} \vee f_{2}\right)$, on the 2complex $K_{1} \vee K_{2}$ so that $\operatorname{pr} \circ\left(f_{1} \vee f_{2}\right) \circ$ incl $=f_{j}$ for $j=1,2$, where pr is the projection map of $S^{1} \vee S^{1}$ onto $S^{1}$. Following is a commutative diagram showing the relationship between these maps.

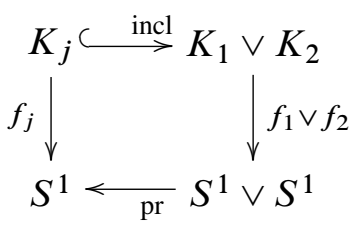

The ascending and descending links of $v$ are each 2-component forests (the union of the ascending and descending links of $v_{1} \in K_{1}$ and $v_{2} \in K_{2}$ ). To complete the 
construction, we attach a single 2-cell which represents a relation $r$ of the form $a_{0}^{ \pm 1} b_{1}^{ \pm 1}=b_{0}^{ \pm 1} a_{1}^{ \pm 1}$ where these are the 1-cells described in hypothesis (iii). The powers in the relation are positive or negative depending upon the sign of the associated $\alpha_{i}, \beta_{i}$. Since $\left|\alpha_{0}\right|+\left|\beta_{1}\right|=\left|\alpha_{1}\right|+\left|\beta_{0}\right|$, we can use Lemma 1.5 of Scott and Wall's paper [14] to extend the Morse function of $K_{1} \vee K_{2}$ over $K$. We will call this new function $g$. The extra 2-cell contributes an edge each to the ascending and descending links connecting up the two components of the forest in each case. Thus the ascending and descending links are now trees and $\pi_{1}(K)$ is free-by-cyclic by Proposition 2.10. The following commutative diagram shows the Morse function on the complex $K$.

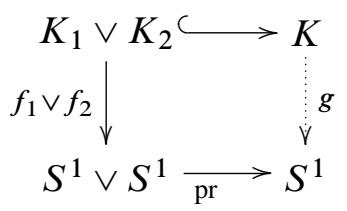

(ii) There is also a map from $K_{1} \vee K_{2}$ to $S^{1} \times S^{1}$ which is shown in the next diagram. Since $K_{1} \vee K_{2}$ is a sub-complex of $K$ we can use the same result as above in [14] and extend this map to a map $g^{\prime}$ over $K$.

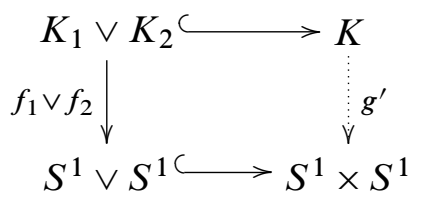

This gives us a map from $\pi_{1}(K)=\left(\pi_{1}\left(K_{1}\right) * \pi_{1}\left(K_{2}\right)\right) /<<r>>$ to $\mathbb{Z}^{2}$. The map to $\mathbb{Z}^{2}$ is onto since it is composed of $f_{1_{*}}: \pi_{1}\left(K_{1}\right) \rightarrow \mathbb{Z}$ and $f_{2_{*}}: \pi_{1}\left(K_{2}\right) \rightarrow \mathbb{Z}$, where the maps $f_{j_{*}}$ are defined by sending generators of $\pi_{1}\left(K_{j}\right)$ to generators of each factor of $\mathbb{Z}$.

Now all the pieces are in place for us to provide the proof of the main theorem.

Proof of Theorem 1.1 We begin with two copies of our LOT group namely, $L_{\left(a_{i}\right)}$,

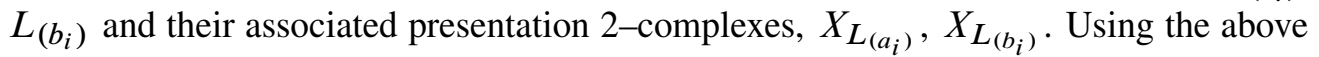
construction, we obtain a new complex $X_{1}$ such that its fundamental group is:

$$
G_{1}=\left(L_{\left(a_{i}\right)} * L_{\left.\left(b_{i}\right)\right)} /\left\langle\left\langle a_{0} b_{2} a_{1}^{-1} b_{0}^{-1}\right\rangle\right\rangle=\left\langle L_{\left(a_{i}\right)}, L_{\left(b_{i}\right)} \mid a_{0} b_{2} a_{1}^{-1} b_{0}^{-1}\right\rangle\right.
$$

Any choice of relation $a_{i} b_{j}=b_{k} a_{l}$ would fit the conditions of Theorem 4.2, but not all of these would ensure that our resultant group is hyperbolic, so we have carefully 
chosen indices for the generators in the relation so that the link of the new complex $X_{1}$ is large and the corresponding universal cover contains no flat planes. Figure 2 represents the link $X_{1}$. As we can see, there are no new circuits of length less than five created in the link when the edges corresponding to the extra square 2-cell are added. Thus, $X_{1}$ is non-positively curved and the poison corners from each original complex are still present in $X_{1}$, prohibiting the development of a flat plane in the universal cover $\tilde{X}_{1}$. Therefore, $\widetilde{X}_{1}$ is $\delta$-hyperbolic and $G_{1}$ is a hyperbolic group. Theorem 4.2 implies our group is free-by-cyclic and there is a map from $G_{1}$ onto $\mathbb{Z}^{2}$, thus completing the proof of the first statement of Theorem 1.1.

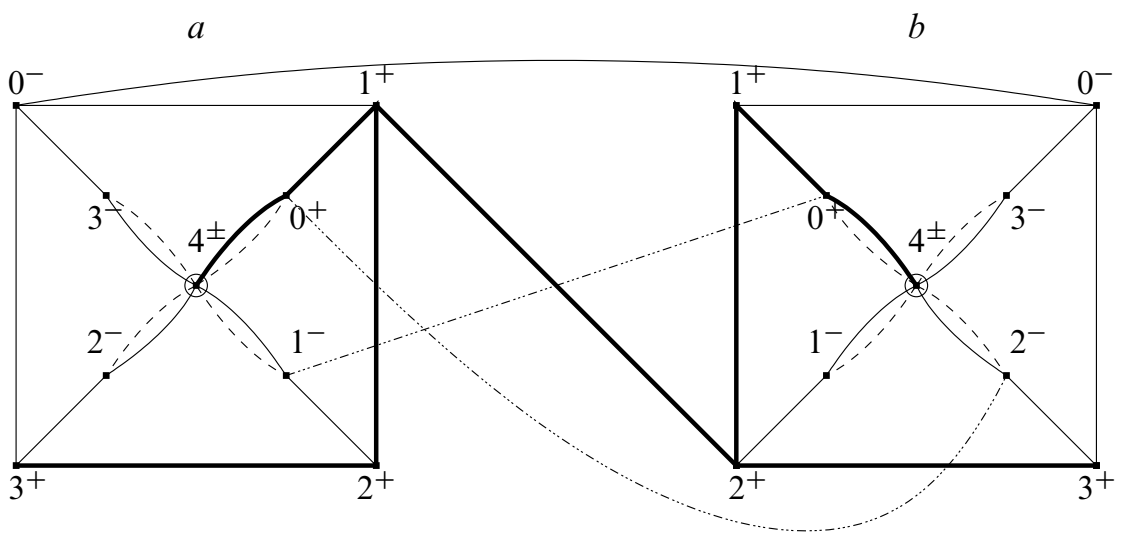

Figure 2: $\operatorname{Lk}\left(v, X_{1}\right)$. The descending link is shown in bold.

We leave it to the reader to check that $\left\langle L_{\left(k_{1}, a_{i}\right)}, L_{\left(k_{2}, b_{i}\right)} \mid a_{0} b_{2} a_{1}^{-1} b_{0}^{-1}\right\rangle$ is also freeby-cyclic and hyperbolic for all $k_{1}, k_{2} \geqslant 4$. The following lemma gives us a formula for calculating the free rank of the kernel when there is an epimorphism from these groups onto $\mathbb{Z}$.

Lemma 4.3 Let $K$ be a 2-complex constructed as in Theorem 4.2 such that $K_{j}=$ $X_{L_{\left(a_{i}, k_{j}\right)}}$ for $k_{1}, k_{2} \geqslant 4$ and the added 2-cell reads $a_{0}^{ \pm 1} b_{2}^{ \pm 1} a_{1}^{\mp 1} b_{0}^{\mp 1}$ around the boundary. Let $f_{j}: K_{j} \rightarrow S^{1}, j=1,2$, be circled-valued Morse functions such that $m=\left|f_{1 *}\left(\left[a_{i}\right]\right)\right|$ and $n=\left|f_{2 *}\left(\left[b_{i}\right]\right)\right|$, and let $g: K \rightarrow S^{1}$ be the circle valued Morse function obtained from the construction in Theorem 4.2. Then, if $m, n \neq 0$ and $\operatorname{gcd}(m, n)=1, \chi\left(g^{-1}(p)\right)=-\left(k_{1} m+k_{2} n\right)$.

Proof First we will examine the point pre-images of the map $f_{j}: K_{j} \rightarrow S^{1}$. Since we are dealing with affine cell-complexes and affine maps, these pre-images are graphs. 

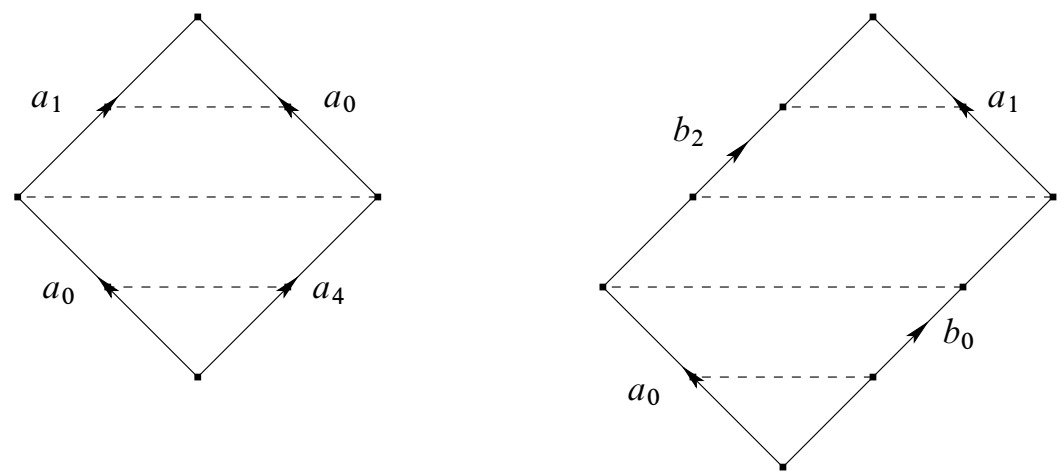

Figure 3: Example 2-cells of $K$ for the case $m=2$ and $n=3$, with the edges sub-divided. The dashed lines represent the pre-image of $p$ in these 2-cells.

Since $K_{j}=X_{L_{\left(a_{i}, k\right)}}, K_{j}$ is made up of $k$ square 2-cells with $(k+1)$ different edge labels. For $\left|f_{j_{*}}\left(\left[a_{i}\right]\right)\right|=m$, the pre-image of the basepoint $p$ of $S^{1}$ is the union of its pre-images in the 2-cells. Each edge will have to be subdivided which will add $(m-1)$ new vertices for each edge label (that is, $(k+1)(m-1)+1$ total vertices). The number of edges in the pre-image in a single 2-cell is equal to the number of pairs of opposing vertices in the $2-$ cell, which is $2(m-1)+1$. The left-hand side of Figure 3 shows a sample cell for the case $m=2$. The resulting graph has Euler characteristic

$$
(k+1)(m-1)+1-k(2(m-1)+1)=-(k-1) m .
$$

Now we will look at the pre-image in the added 2-cell. (The case where $m=2$ and $n=3$ is shown in Figure 3.) We will not count vertices since they are counted in the building block complexes. The number of edges is $(m+n-1)$ which is again the number of opposing pairs of vertices in the cell.

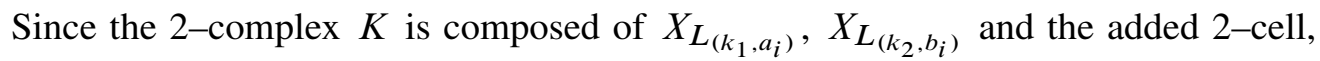
after making the correction for the over count of the original vertex by subtracting 1 , we get:

$$
\chi\left(g^{-1}(p)\right)=-\left(k_{1}-1\right) m+-\left(k_{2}-1\right) n-(m+n-1)-1=-\left(k_{1} m+k_{2} n\right) .
$$

Remark 4.4 For $\operatorname{gcd}(m, n)=d>1$ let $m^{\prime}=m / d, n^{\prime}=n / d$; then $\operatorname{gcd}\left(m^{\prime}, n^{\prime}\right)=1$. Using Lemma 4.3 we have $\chi\left(h^{-1}(p)\right)=1-d\left(k_{1} m^{\prime}+k_{2} n^{\prime}\right)-d$ where $h$ is the composition of the circle-valued Morse function $g$ in the lemma above with a map which is multiplication by $d$ at the fundamental group level, that is, $h_{*}=(\times d) \circ g_{*}$. 
Remark 4.5 From the above result we see that the free rank of any group constructed in this manner is $\left(k_{1} m+k_{2} n+1\right)$. The minimal free rank of $G_{1}$ above is therefore 9, and using these methods, we can construct a group with any minimal free rank $k \geqslant 9$.

Finally we claim that the examples we obtained in this section so far have reducible automorphisms associated with them. Bestvina and Handel [2] gave the following definition of reducible and irreducible automorphisms.

Definition 4.6 An automorphism of a group $\mathrm{G}$ is reducible if it preserves the conjugacy class of a proper free factor of $\mathrm{G}$. If the automorphism is not reducible then it is irreducible.

The main example produced in this section is the group $F_{9} \rtimes \mathbb{Z}$. This group was constructed as a one-relator quotient of a free product of two free-by-cyclic groups (both of free rank 4). We will show that there is a reducible automorphism associated with this group.
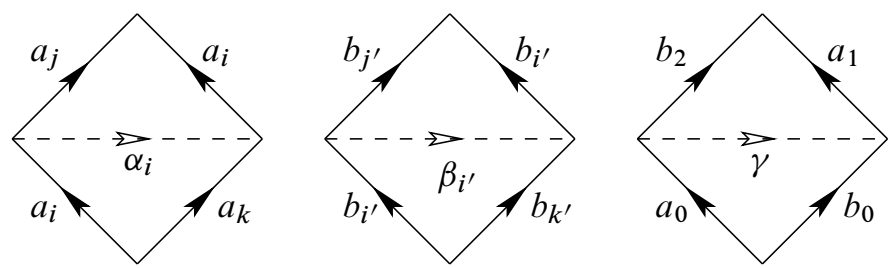

Figure 4: The generators of $F_{9}$. The generators are labeled according to the edge labels of the corresponding 2-cell which appear twice in the cell.

Proposition 4.7 All automorphisms associated with the group $F_{9} \rtimes \mathbb{Z}$ constructed in the example above are reducible.

Proof Consider the pre-image of the base-point of the target circle under the Morse function $g: X \rightarrow S^{1}$ (from Theorem 4.2). This pre-image can be described as a union of the pre-images under $f_{1}: X_{L_{\left(a_{i}\right)}} \rightarrow S^{1}$ and $f_{2}: X_{L_{\left(b_{i}\right)}} \rightarrow S^{1}$ along with the pre-image in the extra 2-cell. The pre-images under $f_{1}$ and $f_{2}$ are bouquets of 4 circles. Let $\alpha_{0}, \ldots, \alpha_{3}$ be the generators of the free group (denoted by $F_{\alpha_{i}}$ ) corresponding to the pre-image under $f_{1}$, similarly let $\beta_{0}, \ldots, \beta_{3}$ be the generators of the free group (denoted by $F_{\beta_{i}}$ ) corresponding to the pre-image under $f_{2}$, and let the generator corresponding to the pre-image in the remaining 2-cell be $\gamma$. Proposition 4.7 shows generic square 2-cells of the 2-complex $X$ and the edge running through the middle of each square $2-$ cell representing the generators $\alpha_{i}, \beta_{i^{\prime}}$ and $\gamma$. 

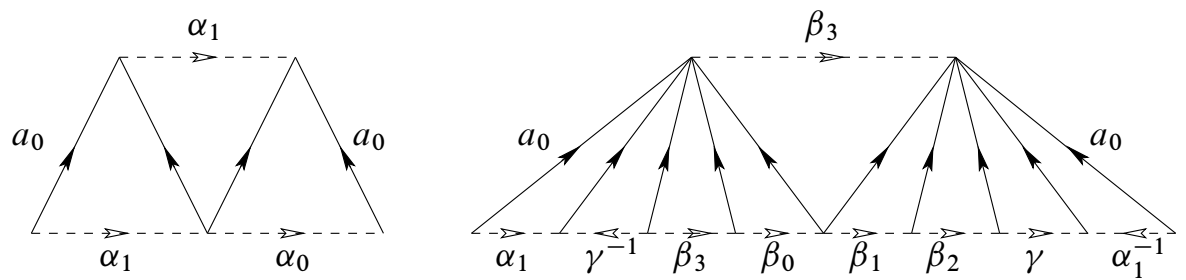

Figure 5: The effect of conjugating by $a_{0}$ on the generators $\alpha_{1}$ and $\beta_{3}$. The automorphism is given by reading the labels on the bottom of the figures.

If we conjugate by a generator from the group $L_{\left(a_{i}\right)}$, say $a_{0}$, then $F_{\alpha_{i}}$ is invariant. This can be shown by examining what effect this conjugation has on the generators.

$$
\begin{aligned}
& a_{0} \alpha_{0} a_{0}{ }^{-1}=\alpha_{3}^{-1} \alpha_{2}^{-1} \alpha_{1}^{-1}, a_{0} \alpha_{1} a_{0}^{-1}=\alpha_{1} \alpha_{0}, \\
& a_{0} \alpha_{2} a_{0}{ }^{-1}=\alpha_{1} \alpha_{2} \alpha_{0}, a_{0} \alpha_{3} a_{0}{ }^{-1}=\alpha_{1} \alpha_{2} \alpha_{3} \alpha_{0}
\end{aligned}
$$

Figure 5 illustrates how these relations are obtained. The automorphism corresponding to the above conjugation actually keeps $F_{\alpha_{i}}$ invariant. Thus this automorphism is reducible. We can also see that $F_{\beta_{i}}$ is not invariant, but it is invariant up to conjugation. Conjugating by $a_{0}$, gives us $\left(\alpha_{1} \gamma^{-1}\right) F_{\beta_{i}}\left(\alpha_{1} \gamma^{-1}\right)^{-1}$ as seen below.

$$
\begin{gathered}
a_{0} \beta_{0} a_{0}^{-1}=\alpha_{1} \gamma^{-1} \beta_{2}^{-1} \beta_{1}^{-1} \beta_{3}^{-1} \gamma \alpha_{1}^{-1}, a_{0} \beta_{1} a_{0}^{-1}=\alpha_{1} \gamma^{-1} \beta_{2}^{-1} \beta_{0} \beta_{1} \beta_{2} \gamma \alpha_{1}^{-1} \\
a_{0} \beta_{2} a_{0}^{-1}=\alpha_{1} \gamma^{-1} \beta_{0} \beta_{1} \beta_{2} \gamma \alpha_{1}^{-1}, a_{0} \beta_{3} a_{0}^{-1}=\alpha_{1} \gamma^{-1} \beta_{3} \beta_{0} \beta_{1} \beta_{2} \gamma \alpha_{1}^{-1}
\end{gathered}
$$

In fact, conjugating by any $a_{i}$ generator will keep $F_{\alpha_{i}}$ invariant just as conjugating by any $b_{i}$ generator will keep $F_{\beta_{i}}$ invariant. If we conjugate by other elements that map to $1 \in \mathbb{Z}$, we will still preserve the conjugacy classes of these factors. For example:

$$
\left(b_{0} a_{1}^{-1} a_{0}\right) F_{\beta_{i}}\left(a_{0}^{-1} a_{1} b_{0}^{-1}\right) \cong\left(b_{0} a_{1}^{-1}\right) \alpha \gamma^{-1} F_{\beta_{i}} \gamma \alpha^{-1}\left(a_{1} b_{0}^{-1}\right)
$$

Since $b_{0} a_{0}^{-1}$ is in the kernel of $g_{*}$, we see that the conjugacy class of $F_{\beta_{i}}$ is still preserved under conjugation in $F_{9} \rtimes \mathbb{Z}$. Thus any automorphism will be reducible. $\square$

This same technique can be used to give reducible automorphisms for any group of the form, $\left\langle L_{\left(k_{1}, a_{i}\right)}, L_{\left(k_{2}, b_{i}\right)} \mid a_{0} b_{2} a_{1}^{-1} b_{0}^{-1}\right\rangle$, for $k_{1}, k_{2} \geqslant 4$.

\section{A Labeled Oriented Forest example}

By construction, the examples produced in the previous section are necessarily reducible. In this section we will use a different method of construction to produce an example 
that does not immediately appear to be reducible. This construction is similar to that presented in Section 4, but instead of taking a free product of two separate groups and adding a relation, we will simply add a relation to an already existing group. The group we will begin with will be defined from a Labeled Oriented Forest (LOF).
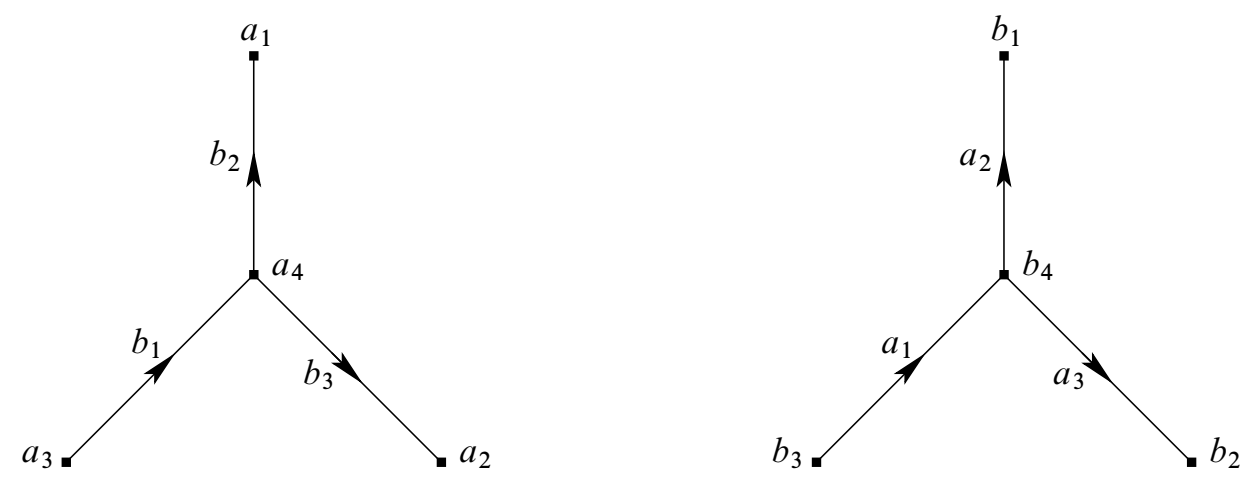

Figure 6: The LOF corresponding to the group $G_{F}$.

Example 5.1 (Labeled Oriented Forest): Our LOF will consist of the two trees shown in Figure 6 . The first tree has vertices labeled by $a_{1}, \ldots, a_{4}$ and the second has vertices labeled by $b_{1}, \ldots, b_{4}$. The edges of each tree are labeled by vertices of the opposite tree. The presentation 2-complex $X$ of this LOF, is comprised of six square 2-cells and admits a circle-valued Morse function $f: X \rightarrow S^{1}$. The ascending and descending links are 2 -component forests. The corresponding group is

$G_{F}=\left\langle a_{i}, b_{i}, i=1 \ldots 4 \mid a_{1}^{b_{2}}=a_{4}, a_{2}^{b_{3}}=a_{4}, a_{4}^{b_{1}}=a_{3}, b_{1}^{a_{2}}=b_{4}, b_{2}^{a_{3}}=b_{4}, b_{4}^{a_{1}}=b_{3}\right\rangle$.

The link of the complex (see Figure 7) has no bigons or empty triangles, therefore the link is large. No flat planes develop in the universal cover because every 2-cell of our complex has not just one, but two poison corners.

We complete the construction by adding an extra square 2-cell that represents the relation $a_{4} b_{1}=b_{4} a_{1}$. The Morse function $f$ extends to a map $g_{2}$ over the new complex which we will call $X_{2}$. The corresponding group $G_{2}$ is denoted by $\left\langle G_{F} \mid a_{4} b_{1} a_{1}^{-1} b_{4}^{-1}\right\rangle$.

The ascending and descending links corresponding to the new complex are now trees as the new relation contributes one edge to each link joining up the 2-components of each forest. Hence, the corresponding group is free-by-cyclic by Proposition 2.10. The addition of this cell has added length 4 circuits to our link, so we must now check the new link to show that $G_{2}$ is hyperbolic. The new link is shown on the right in Figure 
7. The four edges contributed by the new 2-cell are denoted here by dashed-dotted lines between the following vertices: $b_{4}{ }^{+} a_{1}{ }^{-}, b_{4}{ }^{-} a_{4}{ }^{-}, a_{4}{ }^{+} b_{1}{ }^{-}$, and $a_{1}{ }^{+} b_{1}{ }^{+}$. We can see that where we previously had twelve poison corners, we now have only four. Consequently, our new 2-cell, and two of our original 2-cells no longer have poison corners. The following lemma will show that $G_{2}$ is indeed hyperbolic.
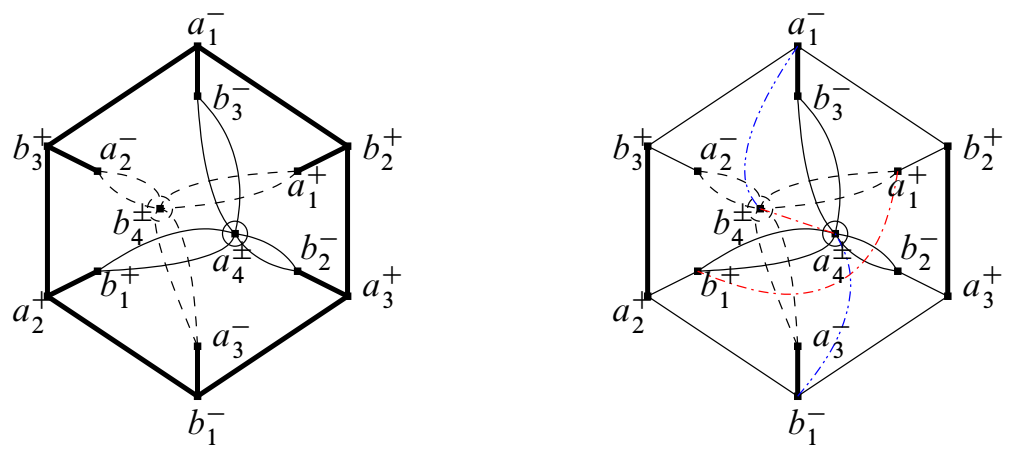

Figure 7: $\operatorname{Lk}(X, v)$ and $\operatorname{Lk}\left(X_{2}, v\right)$. The circles indicate suspension points as in Figure 1. Poison Corners are indicated by bold edges. Note that although the one dot-dash edges and the two dot-dash edges appear to meet at the suspension points, they do not. A careful check shows that there are no circuits of length less than four.

Lemma 5.2 $G_{2}$ is hyperbolic.

Proof In order to prove this lemma, we must check that the universal cover of $X_{2}$ is $\delta$-hyperbolic, that is, we must check that no flat planes can develop in the universal cover $\tilde{X}_{2}$. Any such plane would have to be made up of the new square 2-cell, and only those original 2-cells which now have no poison corners. We will attempt to build a plane starting with the new cell and show that it is impossible to develop a flat plane, thus proving the lemma. The 2-cells available to us are shown at the top of Definition 3.4. In the bottom of Definition 3.4, we see that it does not take long to discover a vertex around which we cannot find a Euclidean neighborhood (indicated by the large arrow).

$G_{2}$ can be mapped onto $\mathbb{Z}^{2}$ because the $a_{i}$ generators and $b_{i}$ generators can be sent independently to generators of $\mathbb{Z}^{2}$. Hence, $G_{2}$ fibers in infinitely many ways over $\mathbb{Z}$. The following lemma investigates the ranks of the kernels of these maps. 

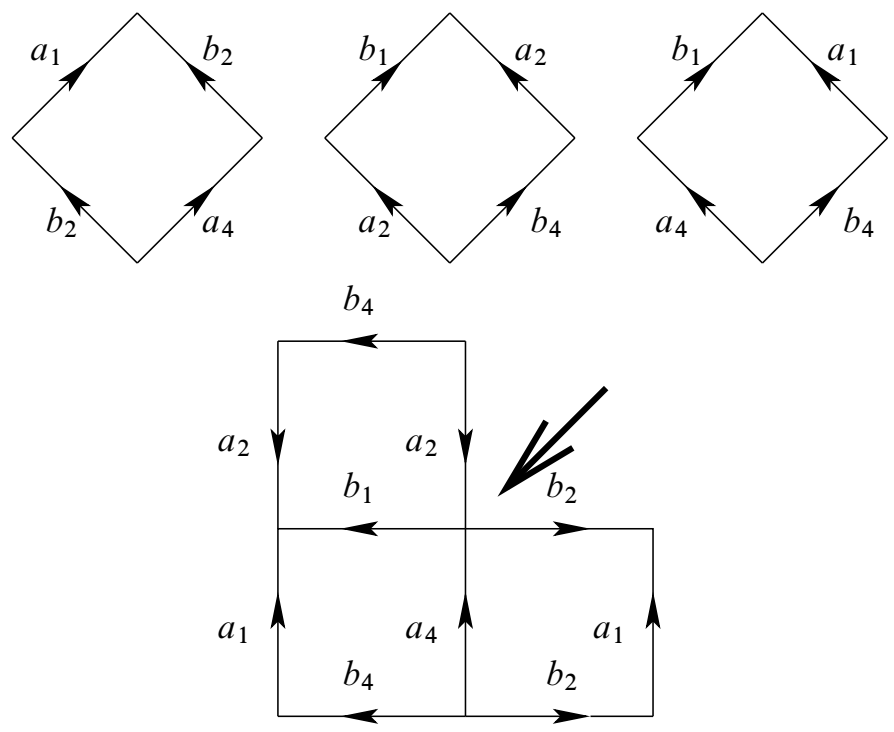

Figure 8: The 2-cells of $X_{2}$ which have no poison corners fail to develop into a plane in the universal cover.

Lemma 5.3 Let $G_{2}$ and $g_{2}$ be as in Example 5.1. Suppose that $m=g_{2 *}\left(\left[a_{i}\right]\right)$ and $n=g_{2 *}\left(\left[b_{i}\right]\right)$ for $i=1, \ldots, 4$. Then if $m, n \neq 0$ and $\operatorname{gcd}(m, n)=1$, the rank of the kernel of the map $g_{2 *}: G_{2} \rightarrow Z$ is given by the following expression: $1+(3|m|+3|n|)$.

Proof By construction, $m>0$ implies $n>0$, so we will assume without loss of generality that $m, n>0$. In order to find the rank, we will examine the Euler characteristic of the graph which is the pre-image of the base-point of $S^{1}$ under the map $g_{2}$. Since the 2-complex contains seven 2-cells, we will look at what the effect of changing the map $g_{2_{*}}$ has on each 2-cell. In the presentation 2-complex, all the 2-cells are basically the same with 2 opposing $a$ edges and 2 opposing $b$ edges. For $m=1$ and $n=1$, the pre-image has one vertex and seven edges (one from each 2-cell), so the rank of the kernel is 7 . Otherwise we must subdivide the edges, adding new vertices. The number of new vertices corresponding to each $a_{i}, b_{i}$ edge is $4(m-1), 4(n-1)$ respectively. The number of edges in each $2-$ cell is $(m+n-1)$ (see the right-hand side Figure 3), so in all there are $7(m+n-1)$ edges. Counting the original vertex, the total number of vertices is $4 m+4 n-7$, so the Euler characteristic of the graph is $-(3 m+3 n)$. Therefore the free rank of the kernel is $1+3 m+3 n$. 
Remark 5.4 For the example constructed in this section, we can show that there is a topological representative of an outer automorphism which is irreducible. We will show this using definitions and techniques from Bestvina-Handel's paper [2, Section 1].

Let $R_{n}$ be the bouquet on $n$-circles. A marked graph $G$ is a graph with a homotopy equivalence $\tau: R_{n} \rightarrow G$. A homotopy equivalence $f: G \rightarrow G$ determines an outer automorphism $\mathcal{O}$, of $F_{n}$. If $V$ is the vertex set of $G$ and $\left.f\right|_{(G \backslash V)}$ is locally injective, then we say that $f: G \rightarrow G$ is a topological representative of $\mathcal{O}$. The transition matrix $M$ for a topological representative $f$ has entries $a_{i j}$ defined as the number of times that the $f$-image of the $j^{\text {th }}$ edge crosses the $i^{\text {th }}$ edge in either direction. A nonnegative integral matrix $M$ is irreducible if for all $1 \leqslant i, j \leqslant \operatorname{dim}(M)$, there exists $N(i, j)>0$ so that the $i j^{\text {th }}$ entry of $M^{N(i, j)}$ is positive. A topological representative, $f$, is irreducible if and only if its transition matrix is irreducible. An outer automorphism $\mathcal{O}$ is irreducible if and only if every topological representative of $\mathcal{O}$ is irreducible.

Consider the map $g_{2 *}: G_{2} \rightarrow \mathbb{Z}$ such that $1=g_{2 *}\left(\left[a_{i}\right]\right), 1=g_{2 *}\left(\left[b_{i}\right]\right)$. Using the same naming convention as in Proposition 4.7, let the generators of the $\operatorname{Ker}\left(g_{2 *}\right)$ be $\alpha_{1}, \alpha_{2}, \alpha_{3}, \beta_{1}, \beta_{2}, \beta_{3}, \gamma$. If we conjugate the generators by $a_{3}$ say, then we have the following expressions:

$$
\begin{gathered}
a_{3} \alpha_{1} a_{3}{ }^{-1}=\alpha_{3}^{-1} \beta_{2}^{-1} \beta_{3}^{-1} \alpha_{2}^{-1} \gamma \beta_{2} \alpha_{3}, a_{3} \alpha_{2} a_{3}{ }^{-1}=\alpha_{3}^{-1} \beta_{2}^{-1} \gamma^{-1} \alpha_{2} \alpha_{1} \beta_{2} \alpha_{3}, \\
a_{3} \alpha_{3} a_{3}^{-1}=\alpha_{1} \beta_{2} \alpha_{3}, a_{3} \beta_{1} a_{3}^{-1}=\alpha_{3}^{-1} \beta_{2}^{-1} \gamma^{-1}, a_{3} \beta_{2} a_{3}{ }^{-1}=\alpha_{3}^{-1} \beta_{1} \gamma \beta_{2} \alpha_{3}, \\
a_{3} \beta_{3} a_{3}{ }^{-1}=\alpha_{3}^{-1} \beta_{2}^{-1} \gamma^{-1} \alpha_{2} \beta_{3} \beta_{1} \gamma \beta_{2} \alpha_{3}, a_{3} \gamma a_{3}{ }^{-1}=\alpha_{3}^{-1} \beta_{2}^{-1} \gamma^{-1} \beta_{1}^{-1} \alpha_{1} \beta_{2} \alpha_{3}
\end{gathered}
$$

This gives us a topological representative of an outer automorphism $\mathcal{O}$ and the corresponding transition matrix $M$ is a $7 \times 7$ nonnegative matrix. Using applicable software, it is easy to calculate the powers of this matrix and see that all entries of $M^{3}$ are positive. Therefore, we have an irreducible topological representative of the outer automorphism; however, using this method it is not possible to check that every topological representative is irreducible. Gersten and Stallings [8] provide a method for showing that an outer automorphism is irreducible, using super irreducible characteristic polynomials of $\mathcal{O}_{a b}$. This method also failed to give a positive result due to the abelianization process involved.

\section{Questions}

Following are some open questions concerning hyperbolic, free-by-cyclic groups that fiber in infinitely many ways. 
Question 1 We know that the minimal free rank of kernels for hyperbolic, free-bycyclic groups is 3 . Using our methods we can generate groups that fiber in infinitely many ways with free kernel of minimal rank 7 and any minimal rank $k \geqslant 9$. Brady and Crisp produced a family of groups in [3] which will also fiber in infinitely many ways with free kernel of minimal rank 6. Can we find lower rank examples that fiber in infinitely many ways?

The impetus for this research was work done in the 3-manifold setting. The next question relates to results obtained in that setting.

Question 2 Every hyperbolic 3-manifold that fibers in infinitely many ways has an irreducible outer automorphism. Is the automorphism in the LOF example irreducible? Are there any other examples with irreducible automorphisms?

All of our examples have maps onto $\mathbb{Z}^{2}$. A group is said to be large if a finite index subgroup maps onto $F_{2}$. Our next question deals with possible connections to large groups.

Question 3 Are there free-by-cyclic groups that fiber in only one way, such as $L_{\left(a_{i}\right)}$, which have finite index subgroups which fiber in infinitely many ways? Will these groups have infinite virtual first Betti number? Would these groups be large?

\section{References}

[1] J Barnard, N Brady, Distortion of surface groups in CAT(0) free-by-cyclic groups, Geom. Dedicata 120 (2006) 119-139 MR2252898

[2] M Bestvina, M Handel, Train tracks and automorphisms of free groups, Ann. of Math. (2) 135 (1992) 1-51 MR1147956

[3] N Brady, J Crisp, CAT(0) and CAT(-1) dimensions of torsion free hyperbolic groups, Comment. Math. Helv. 82 (2007) 61-85 MR2296058

[4] M R Bridson, On the existence of flat planes in spaces of nonpositive curvature, Proc. Amer. Math. Soc. 123 (1995) 223-235 MR1273477

[5] M R Bridson, A Haefliger, Metric spaces of non-positive curvature, volume 319 of Grundlehren der Mathematischen Wissenschaften [Fundamental Principles of Mathematical Sciences], Springer, Berlin (1999) MR1744486

[6] J O Button, Mapping tori with first Betti number at least two, J. Math. Soc. Japan 59 (2007) 351-370 MR2325689

[7] P Eberlein, Geodesic flow in certain manifolds without conjugate points, Trans. Amer. Math. Soc. 167 (1972) 151-170 MR0295387 
[8] S M Gersten, J R Stallings, Irreducible outer automorphisms of a free group, Proc. Amer. Math. Soc. 111 (1991) 309-314 MR1052571

[9] M Gromov, Hyperbolic groups, from: "Essays in group theory", Math. Sci. Res. Inst. Publ. 8, Springer, New York (1987) 75-263 MR919829

[10] H M Hilden, M T Lozano, J M Montesinos-Amilibia, On hyperbolic 3-manifolds with an infinite number of fibrations over $S^{1}$, Math. Proc. Cambridge Philos. Soc. 140 (2006) 79-93 MR2197577

[11] J Howie, On the asphericity of ribbon disc complements, Trans. Amer. Math. Soc. 289 (1985) 281-302 MR779064

[12] I J Leary, G A Niblo, D T Wise, Some free-by-cyclic groups, from: "Groups St. Andrews 1997 in Bath, II”, London Math. Soc. Lecture Note Ser. 261, Cambridge Univ. Press, Cambridge (1999) 512-516 MR1676647

[13] D A Neumann, 3-manifolds fibering over $S^{1}$, Proc. Amer. Math. Soc. 58 (1976) 353-356 MR0413105

[14] P Scott, T Wall, Topological methods in group theory, from: "Homological group theory (Proc. Sympos., Durham, 1977)", London Math. Soc. Lecture Note Ser. 36, Cambridge Univ. Press, Cambridge (1979) 137-203 MR564422

[15] J Stallings, On fibering certain 3-manifolds, from: "Topology of 3-manifolds and related topics (Proc. The Univ. of Georgia Institute, 1961)”, Prentice-Hall, Englewood Cliffs, N.J. (1962) 95-100 MR0158375

[16] WP Thurston, Hyperbolic structures on 3-manifolds II: Surface groups and 3manifolds that fiber over the circle arXiv:math.GT/9801045

[17] J L Tollefson, 3-manifolds fibering over $S^{1}$ with nonunique connected fiber, Proc. Amer. Math. Soc. 21 (1969) 79-80 MR0236945

Department of Mathematics, College of Mount St. Joseph, 5701 Delhi Road Cincinnati, Ohio 45233-1670, USA

Department of Mathematics and Computer Science, The Citadel, 171 Moultrie St., Charleston, SC 29409, USA

TaraLee_Mecham@mail.msj.edu, antara.mukherjee@citadel.edu

Received: 20 December 2008 